\title{
How can we reduce the impact of COVID-19 pandemic on timely access to liver transplantation in children?
}

\author{
Mettu Srinivas Reddy ${ }^{1}$ (I) Jagadeesh Menon ${ }^{1} \cdot$ Abdul Rahman Hakeem $^{1} \cdot$ Shivakumar Murugesan $^{1}$. \\ Gomathy Narasimhan ${ }^{1} \cdot$ Naresh Shanmugham ${ }^{1} \cdot$ Mohamed Rela $^{1}$
}

Received: 6 August 2020 / Accepted: 22 December 2020 / Published online: 27 January 2021

(c) Asian Pacific Association for the Study of the Liver 2021

The impact of the COVID-19 pandemic on access to pediatric liver transplantation (LT) is likely to be substantial due to a disproportionate reduction in pediatric deceased donors and a disinclination to split liver grafts during a pandemic. Pediatric living donor liver transplantation (LDLT) is an attractive alternative in this setting. However, COVID-19 related concerns, restrictions on people movement and reduced elective clinical activity can disrupt timely access to pediatric liver care and liver transplant.

The COVID pandemic in India had a delayed start compared to Europe and USA and caseload started increasing after April 2020. A COVID-free pathway for managing children with decompensated liver disease, recipient and donor evaluation and performing pediatric LDLT was established in our unit from May 2020. We assessed the impact of COVID on pediatric referrals and LDLT performed in our unit during the pandemic.

We reviewed data for all children $(<12$ years) referred to our unit for LT during a 6-month period between 1st December 2019 and 30th May 2020 (2020 era). Data were compared with the same time period in 2018-2019 (2019 era).
Number of referrals for LT, PELD score at referral, time to LDLT and reasons for delay were recorded. Children were followed until transplant or death and data was censored till 15th July of the respective years. Each era was divided into three bimonthly intervals (Dec-Jan, Feb-March, April-May) for comparison.

Twenty-eight and 30 children were referred for LT in 2019 and 2020 eras. Number of referrals reduced during April-May 2020 though the difference was not statistically significant (Fig. 1a). However, children referred during April-May 2020 were sicker with higher PELD scores than the contemporaneous 2019 cohort (Median PELD 28 vs 13.1, $p=0.005$ ) (Fig. 1b, Supplementary table). LDLT was performed in $19(67.8 \%)$ and $23(76.7 \%)$ of referred children by the cut-off date in the 2019 and 2020 eras (Fig. 1c; Logrank test; $p=0.891)$. However, time taken from referral to LDLT increased from $33 \pm 19$ days in 2019 to $52 \pm 36$ days in $2020(p=0.039)$. (Fig. 1c, Supplementary table). PostLDLT 30-day survival was similar between 2019 and 2020 eras $(92.3 \%$ vs $100 \%)$.
Supplementary Information The online version contains supplementary material available at https://doi.org/10.1007/s1207 2-020-10128-9.

Mettu Srinivas Reddy

smettu.reddy@gmail.com

1 Institute of Liver Disease and Transplantation, Dr Rela Institute and Medical Center, 7 Works Road, Chromepet, Chennai, India 600044 

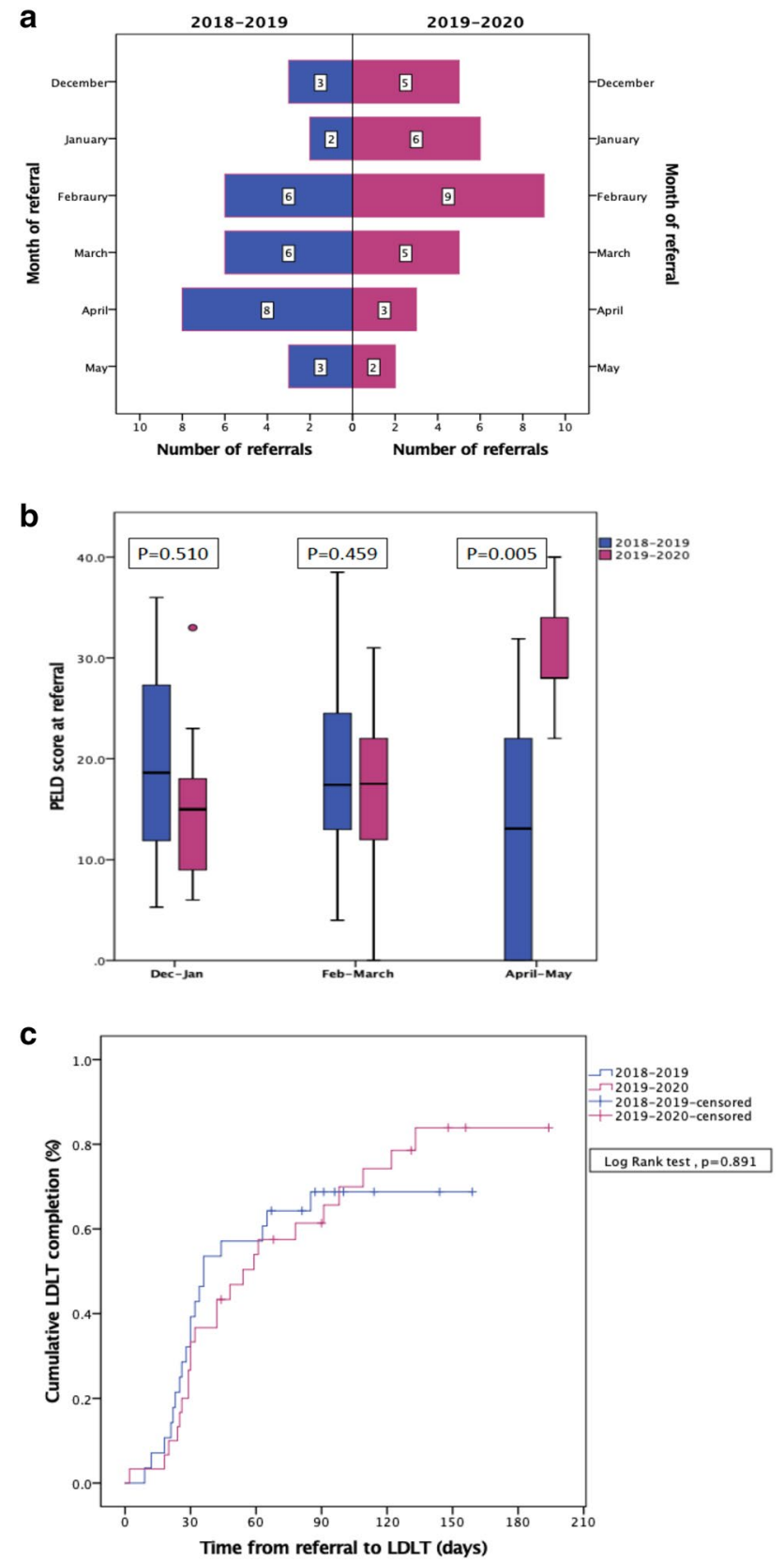

Fig. 1 a Comparison of monthly pediatric LT referrals during 2019 and 2020 eras. b Comparison of PELD scores at referral in 2019 and 2020 in the three bi-monthly periods. c Comparison of time to LDLT for children referred in 2019 and 2020 eras. Data censored until 15th July 2019 and 15th July 2020 for the 2019 and 2020 eras, respectively
Timely LDLT could not be performed in two children due to COVID-lockdown related delays in completing legal documentation. Both children died waiting for LDLT (44 and 64 days after referral). LDLT for one child with acuteon-chronic liver failure was delayed due to SARS-nCOV-2 infection in the donor. One child transplanted in May 2020 was readmitted 2 months post-transplant with SARSnCOV-2 infection and is currently recovering. LDLT was postponed in 5 children as the children were stable and the family elected to wait until the COVID situation improves.

Our data shows that while pediatric referrals for LT reduced during the COVID-19 pandemic, children referred during this period were sicker, probably reflecting a level of selective referral during this period. Timely pediatric LDLT could be safely performed for most children via a COVIDfree clinical pathway. Increased use of electronic documentation and remote authentication may avoid logistic delays to performing LDLT for these sick children.

Publisher's Note Springer Nature remains neutral with regard to jurisdictional claims in published maps and institutional affiliations. 